\title{
Using finger for touchscreen devices; is it safe?
}

\author{
Atieh Merikh Nejadasl \\ Aetrospace Engineering Department, \\ Sharif University of Technology, \\ Tehran, Iran \\ At.merikh@alum.sharif.edu
}

\author{
Mahmoud Chizari \\ School of Engineering and Computer Sciences, \\ University of Hertfordshire, \\ Hatfield, Unitd Kingdom \\ https://orcid.org/0000-0003-0555-1242
}

\begin{abstract}
The use of touchscreen devices become a part of people daily life. Finger is used to communicate with such devices. This paper investigated the interaction between human finger and touch screen devices. A series of experiments was performed to investigate the participants' finger functionality when they press their finger on a touch screen device. The study was concluded that the pain threshold is higher than normal pressing force but for repetitive pressing the level of pain threshold will decrease.
\end{abstract}

Keywords - finger, touch screen, pressing force, pain threshold

\section{INTRODUCTION}

The hand and fingers are the main tool adopted to interact with the external environment. The human hand is highly versatile and prone to risk of disorders because of repetitive interaction like keyboarding and touching touchscreen devices. These repetitive motions can cause musculoskeletal problems in fingers. Many investigations have addressed the issue, and all of these tried to simulate the condition of tapping and touching touchscreens to investigate the amount of the repetitive forces that applied on human finger to understand the initiation and development of pain in fingers. Wu et al. [1] have done an investigation to understand the time dependent muscle force during tapping. In their studies they have simulated time dependent dynamic loading in muscle during the tapping. In parallel, Hu et al. [2] have investigated the effects of the finger extensor mechanism on the finger joints. They have focused on the bone-to-bone contact forces at the interphalangeal and metacarpal joints and the forces in the intrinsic and extrinsic muscles while pressing the finger on a surface or touch screen. Asakawa et al. [3] stablished a touchscreen actuator to apply finger force to the touchscreen device. Although, the amount and direction of the force was unknown, but their aim was to characterize the magnitude, direction and impulse of the force applied during single finger tapping.

Beside all of these studies, there are huge amount of experiment which is designed to simulate human computer interaction to be able to measure the amount of force and the pressure applies on the human fingers as a result of a touch. For example, in a research, Asakawa and co-authors [3] tried to characterize the magnitude, direction and impulse of a force applied during single or multiple finger tapping by designing an experimental setup. While, Savioz and co-authors [4] tried to address the design of a novel computer keyboard by embedding many miniature actuators on buttons to provide a programmable force feedback in response to human strokes. Based on finding of the current study; the force needed to activate a capacitive touch screen, which is currently the most used in smartphones, is of $0,068 \mathrm{~N}$ to $0,1 \mathrm{~N}$ and dwell time of a finger in a touch zone can be less than $100 \mathrm{~ms}$. Although, this may require a catch-and-release process to activate finger from extension to flexion or vis versa. The concern is on the level of the force needed to overcome the catching flexors before a trigger finger happen [5].

This study focuses on the biomechanics of a finger interactions with a touch screen surface. Analysing level of the force at the contact zone between the fingertip and the screen is the objective of the study. The study also investigates the effect of repetitive finger pressing on the finger structure. The position of a finger component extended to press a touchscreen surface and its anatomy has been illustrated in Figure 1.

(a)

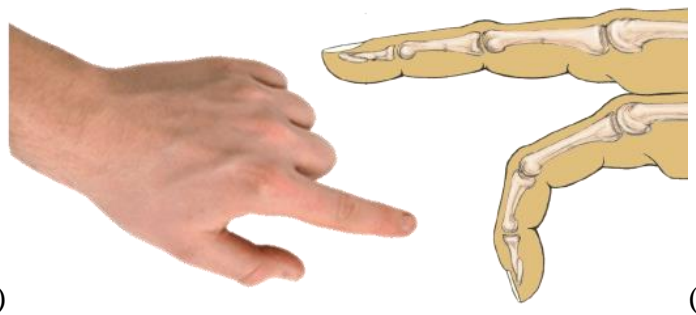

(b)

Figure 1. Index finger in pressing status (a) finger anatomy at extension and flexion status (b)

\section{METHODS}

A series of experiment was setup to assess the amount of force applies to the finger while tapping a touchscreen. The experiment performed using subject valuators from different sex and age group. A local ethical policy was followed to complete the experiment. The intention was to measure the level of force spent on a single or repetitive finger while pressing a touchscreen.

\section{A. Experimental setup; single pressing}

To evaluate the effects of a touch on the finger an experiment was setup. The experiment aimed to measure a maximum pressing force which can be applied by the finger 
before initiating a pain on the finger. To measure the pain threshold on subject patients, an algometer device, as shown in Figure 2, was used. The algometer device is able to identify pressure-pain threshold when the its button pressed by a finger. To maintain a reliable process in the current pressure-pain threshold study, the pressing rate kept constant during the experiment. In the experiment, the subject person was asked to press the algometer button until feel a pain. As the person feel a pain, he/she should release the button and terminate the experiment. When the button was released, then the device measures the pain threshold. This experiment was repeated three times using the index and thumb fingers. The experiment was recorded using 15 subject participants from different sex and age groups.

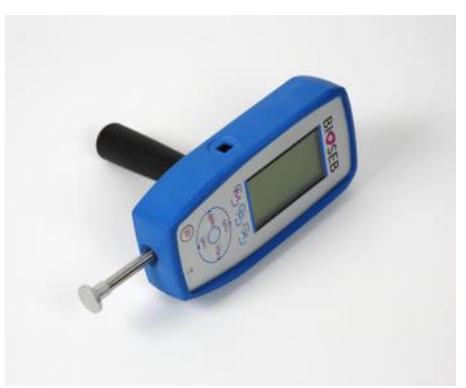

Figure 2. Algometer used for the single pressing experiment. The experimet was repeated three times to obtain reliable results.

\section{B. Results of single pressing test}

The experiment was conducted using 15 subject volunteers from different sex and age groups. The force threshold for each person was recorded using index and thumb fingers. The result of the measurement has been summarized in Table 1 .

TABLE 1. CONTINUES PRESSING EXPERIMENTAL RESULTS PERFORMED ON 15 SUBJECT VOLUNTEERS. IN EACH TEST INDEX AND THUMB FINGERS BEEN TESTED SEPRATLY.

\begin{tabular}{|l|l|l|l|}
\hline Age & Sex & Index finger $(\mathrm{N})$ & Thumb finger $(\mathrm{N})$ \\
\hline 50 & F & 16.93 & 30.7 \\
\hline 56 & F & 37.45 & 46.7 \\
\hline 62 & M & 44.45 & 50.6 \\
\hline 28 & M & 31.60 & 50.7 \\
\hline 18 & F & 26.97 & 30.4 \\
\hline 25 & F & 37.34 & 35.7 \\
\hline 20 & F & 28.64 & 36.7 \\
\hline 53 & M & 33.24 & 40.5 \\
\hline 29 & M & 33.14 & 51.2 \\
\hline 29 & M & 37.08 & 48.7 \\
\hline 28 & M & 36.50 & 50.6 \\
\hline 47 & F & 25.02 & 31.8 \\
\hline 27 & F & 26.84 & 35.3 \\
\hline 24 & F & 21.68 & 32.0 \\
\hline 25 & F & 32.79 & 49.7 \\
\hline
\end{tabular}

\section{Experimental setup; repetitive pressing}

An experiment was setup to measure the cyclical finger pressing threshold. The subject person was asked to toggle the sensor for 100 times simulating texting 100 characters on a touchscreen device. This experiment was repeated with a different age group of volunteers. As a condition of the test, the participants were asked to put her/his finger on a loadcell, as shown in Figure 3, and try to insert similar force as she/he write a text a touchscreen device. The participant was asked to simulate a finger pressing on the loadcell assuming he/she is texting 100 words. During the experiment, a loadcell was recording the inserted force. Figure 4 shows the experimental setup. The custom-made setup was included with a loadcell, an amplifier (Hx-711) and an Arduino board. The device was connected onto a computer to process the data. The experiment was carried out with 3 people. The physical appearance and age of the participant was close. The participant was asked to carry on typing 100 words on the loadcell before they feel pain on their finger. The data was then processed on computer and the amount of force of each tapping was recorded. For each participant the tapping times before feel pain was also recorded.

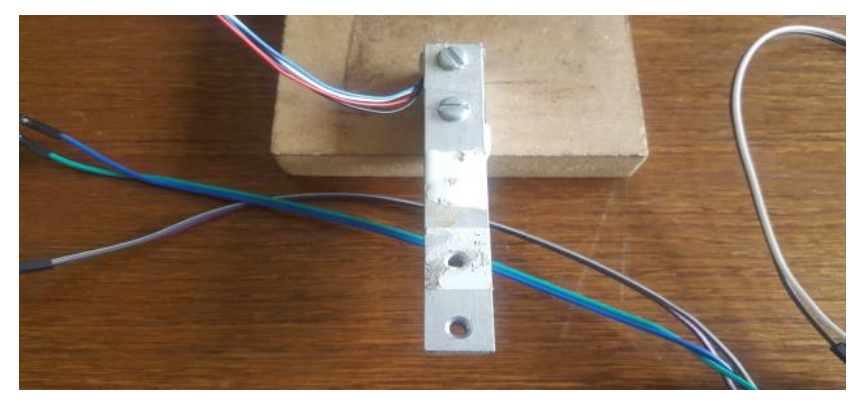

Figure 3. Loadcell for applying force by applicants. The participant was asked to tap 100 times on the loadcell.

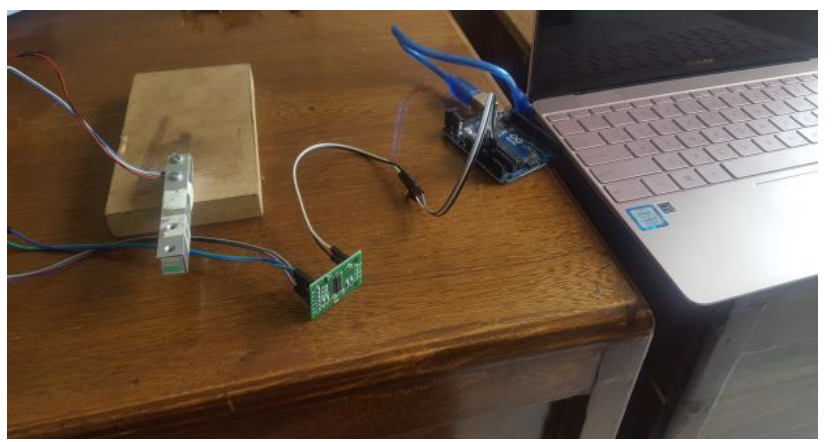

Figure 4. Setup used for repetitive pressing test. The custom-made setup was included with a loadcell, an amplifier $(\mathrm{Hx}-711)$ and an Arduino board.

During the experiment, the captured data by loadcell was sending to the amplifier to amplify the data and send it to the Arduino board. The board then made the data ready to be transferred to the computer. A software on computer was then procesed the data and stored it in an Excel file.

\section{Results for repetitive pressing}

The experiment was focusing on measurement of tapping effects on participants' finger. An interesting outcome was on force distribution on the loadcell. During the experiment 
participant was asked to press his/her finger on the loadcell to simulate tapping on a touchscreen device. Most of the participant felt pain after hundred times repetitive taping. The results confirmed when the pain initiates on the participant finger, the level of force decreased on the loadcell. This effect can be seen on the result plotted in Figure 5. The three participants in this experiment been young with close age and similar physical appearance. As a condition for this experiment, the finger size and skeleton structure of the participants was very similar.

As mentioned earlier, during the experiment each participant was trying to simulate the writing of a 100 word on the loadcell. This was taking between 10 to 15 minutes. It was observed after about 100 tapping on the loadcell the sign of tiredness was appearing on the participants finger and the reaction become slower until the participants feel a pain and terminating the test.

Figure 5 compares the result obtained from the experimental test. Each participant has repeated the tests three times and average result was plotted. By close look at the Figure 5, it can be seen that the maximum pressing force mainly applied at start of the process. The inserted force then become constant until a pain appears on his/her finger. When the pain starts, the pressing force decreases. This shows the effect of repetitive pressing/clicking on the finger.

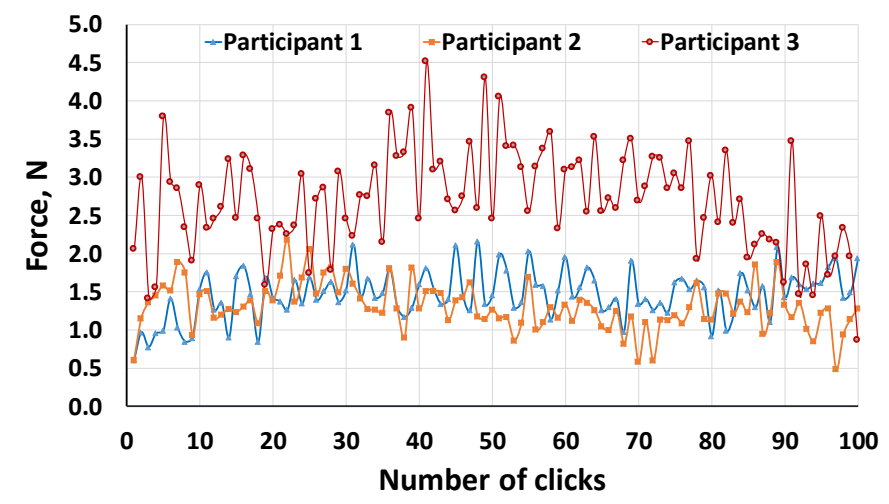

Figure 5. Comparison of pressing force vs. number of click. Three young participants with close age and similar physical appearance carried out the test. All participant been female. Paricipant 1 was 20y/o, parricpan 2 was 20 y/o; paricipant 3 was 18 y/o.

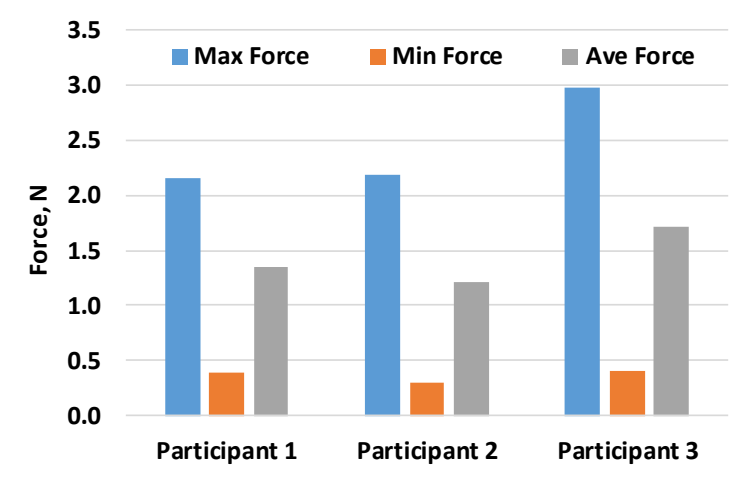

Figure 6. Result of maximum, minimum, average inserted force applied on the loadceel by the three participants.
There are no significant differences between results. Although, three participants are in similar physical appearance, but the result of third participant is slightly different. The results for participant 1 and 2 (both 20 years old) are very close but for the third participant (18 years old), the inserted force is higher. The outcome result of maximum, minimum, average inserted force has been illustrated in Figure 6. Although, the third participant was applying more force on the loadcell at the beginning but at the end, her finger tiredness made her to insert a lower force on the loadcell.

\section{CONCLUSION}

This paper investigated the interaction between human finger and touch screen devices. A series of experiments were performed to investigate the participants' finger functionality when they press their finger on a touch screen device. Following results were concluded from the experiments:

- The pain threshold is higher than normal pressing force. However, in repetitive pressing the level of pain threshold will decrease.

- Younger people apply higher pressing force with bigger force deviation compare to older people.

- Age factor is was shown to be effective on this interaction. As age increases, the inserted force would decrease.

The result of this investigation may help to reduce occupational-related hand disorders by optimizing the design of touch screen devices. Furthermore, the outcome may be used to design a pain relief model for touchscreen users. Further investigation needed to investigate musculoskeletal disorders which may occurs with the excessive use of finger on touchscreen devices.

\section{ACKNOWLEDGEMENTS}

This study is a part of ongoing project. The authors appreciate the generous support received from the Mechanical Engineering Department at Sharif University of Technology on the current research. Kind support received from Mrs Narimani head of Applied Electronics Lab is highly appreciated. Finally, kind support of all participants who helped to measure the experimental data is highly appreciated.

\section{REFERENCES}

[1] Wu JZ, An KN, Cutlip RG, Krajnak K, Welcome D, Dong RG. (2008). Analysis of musculoskeletal loading in an index finger during tapping. J Biomech. 2008;41(3):668-76. Epub 2007 Nov 7.

[2] Hu D, Howard D, Ren L (2014) Biomechanical Analysis of the Human Finger Extensor Mechanism during Isometric Pressing. PLoS ONE 9(4): e94533. https://doi.org/10.1371/journal.pone.0094533

[3] Asakawa DS, Crocker GH, Schmaltz A, Jindrich DL. (2017). Fingertip forces and completion time for index finger and thumb touchscreen gestures. J Electromyogr Kinesiol. 2017 Jun;34:6-13. doi: 10.1016/j.jelekin.2017.02.007. Epub 2017 Feb 24.

[4] G. Savioz, M. Markovic, and Y. Perriard, (2011), Towards multi-finger haptic devices: A computer keyboard with adjustable force feedback, in Electrical Machines and Systems (ICEMS), 2011 International Conference on, pp. 1-6, aug.

[5] Szu-Ching Lu, Li-Chieh Kuo, I-Ming Jou, Chih-Chung Wu, Wen-Lin Tung, (2013) Quantifying Catch-and-Release: The Extensor Tendon Force Needed to Overcome the Catching Flexors in Trigger Fingers, j.ortopaedic research, v31-7, pp 1130-1135 